\title{
Größeneffekte in der Mikrologistik
}

\author{
PROF.DR.-ING. RAINER BRUNS, \\ DIPL.-WI.-ING. GUIDO GRAVENKÖTTER \\ INSTITUT FÜR KONSTRUKTIONS- UND FERTIGUNGSTECHNIK, PROFESSUR FÜR MASCHINENELEMENTE UND TECHNISCHE \\ LOGISTIK, HELMUT-SCHMIDT-UNIVERSITÄT
}

\section{Zusammenfassung}

Obwohl die Mikrosystemtechnik (MST) als „Schlüsseltechnologie des 21. Jahrhunderts“ bezeichnet wird, bleibt der erwartete Durchbruch noch aus. Der vorliegende Artikel geht auf die Herausforderungen für die technische Logistik ein, die mit dem Herunterskalieren der Bauteildimensionen einhergehen. Darüber hinaus werden die Ergebnisse einer Studie präsentiert, die der Lehrstuhl MTL in Unternehmen der MST durchgeführt hat, um die vorherrschende Materialflusstechnik genauer zu untersuchen und Defizite aufzudecken.

\begin{abstract}
Although the micro system technology (MST) is considered the "key technology of the 21st century" it has still failed to achieve a breakthrough. The present article describes the challenges caused by skaleddown component dimensions which technical logistics has to face. Furthermore, it presents the results of a study which the university chair MTL has carried out in various MST companies to analyse the existing material flow technology and to identify deficites.
\end{abstract}

\section{Einleitung}

Die Mikrosystemtechnik (MST) vereint als Querschnittstechnologie elektrische, mechanische, optische, chemische und biologische Funktionen. Typische Anwendungsfelder sind die Medizin- und Life ScienceTechnik, die MST-Branche mit Lieferungen untereinander, sowie insbesondere in Deutschland traditionell die Automobil- bzw. Automotiveindustrie. Für diese Branchen gilt die Mikrosystemtechnik als starker Innovationstreiber. So hat beispielsweise ein moderner PKW zwischen 70 und 100 Sensoren (Airbag, ABS, Ölstand, Neigungsverstellung der Frontlampen u.a.) [Mescheder04].

Erst die vermehrte Integration von Mikrosystemen, auch in Produkten des täglichen Lebens, führt zu einer Erhöhung der Stückzahl und damit auch, aufgrund des Kostendrucks, zu einer verstärkten Forderung nach Automatisierbarkeit der Fertigung und Montage. Vorraussetzung dafür ist jedoch, dass alle im Wertschöpfungsprozess beteiligten Unternehmen die logistischen Rahmenbedingungen für eine Massenproduktion von Mikrosystemen schaffen.

Während die Fertigungstechnik für die Mikrosystemtechnik in der Vergangenheit kontinuierlich weiterentwickelt wurde und im Fokus der Bemühungen stand, so wurde die (technische) Logistik zunächst einmal vernachlässigt. Für die technische Logistik hat der Übergang in die Mikroebene hingegen erst ausschnittsweise - für ausgewählte Teilbereiche - begonnen.

Nachdem das Potential, welches in der Mikrosystemtechnik steckt in Kapitel 2 verdeutlicht wird, geht Kapitel 3 auf die wesentlichen Erkenntnisse einer Studie ein, die am Institut für Maschinenelemente und Technische Logistik durchgeführt worden ist. Sie liefert wertvolle Erkenntnisse auf die Frage, welche Materialflusstechnik in Unternehmen der MST zum Einsatz kommt, und wie sie unter den Einfluss von Größeneffekten eingesetzt wird, sowie die daraus resultierenden Herausforderungen für die technische Logistik („Mikrologistik“). Dazu wurde der Materialfluss verschiedener Produktionsprozesse aufgezeichnet und auf die eingesetzte Technik hin untersucht.

\section{Die Mikrosystemtechnik als Innovationstreiber}

\subsection{Definitionen}

Es existieren unterschiedlich weit gefasste Definitionen zum Begriff Mikrosystemtechnik. Zunächst sollen die am häufigsten verwendeten Definitionen kurz vorgestellt werden. 
Hesselbach definiert die Mikrosystemtechnik als eine „funktionale Integration mechanischer, elektronischer, optischer und sonstiger Funktionselemente unter Anwendung von speziellen Mikrostrukturierungstechniken“ [Hesselbach02].

Allgemeiner gefasst wird der Begriff durch Gerlach und Dötzel: „Die Mikrosystemtechnik umfasst den Entwurf, die Fertigung und die Applikation von miniaturisierten technischen Systemen, deren Elemente und Komponenten typische Strukturgrößen im Mikrometer- und Nanometerbereich besitzen.“ [Gerlach97]. Mescheder wird noch deutlicher und spricht für die Dimension des Mikrobereichs von 0,1 $\mu \mathrm{m}$ bis $100 \mu \mathrm{m}$, wobei die zwei verbleibenden Dimensionen im Millibereich, $100 \mu \mathrm{m}$ bis $10 \mathrm{~mm}$, liegen.

Nach Bark ist ein mikrosystemtechnisches Produkt „durch die Integration von Sensorik, Aktorik und Signalverarbeitung in einem Bauteil sowie durch Baugrößen, die sich zwischen der Halbleitertechnik und der klassischen Feinmechanik befinden“, gekennzeichnet (vgl. Abb.1) [Bark99]. Menz fasst die Aussagen zusammen und postuliert schließlich: „Die Mikrosystemtechnik kann als die konsequente Weiterentwicklung der Mikroelektronik auf nicht elektronische Gebiete angesehen werden.“ [Menz05].

\section{Mikrosystem}

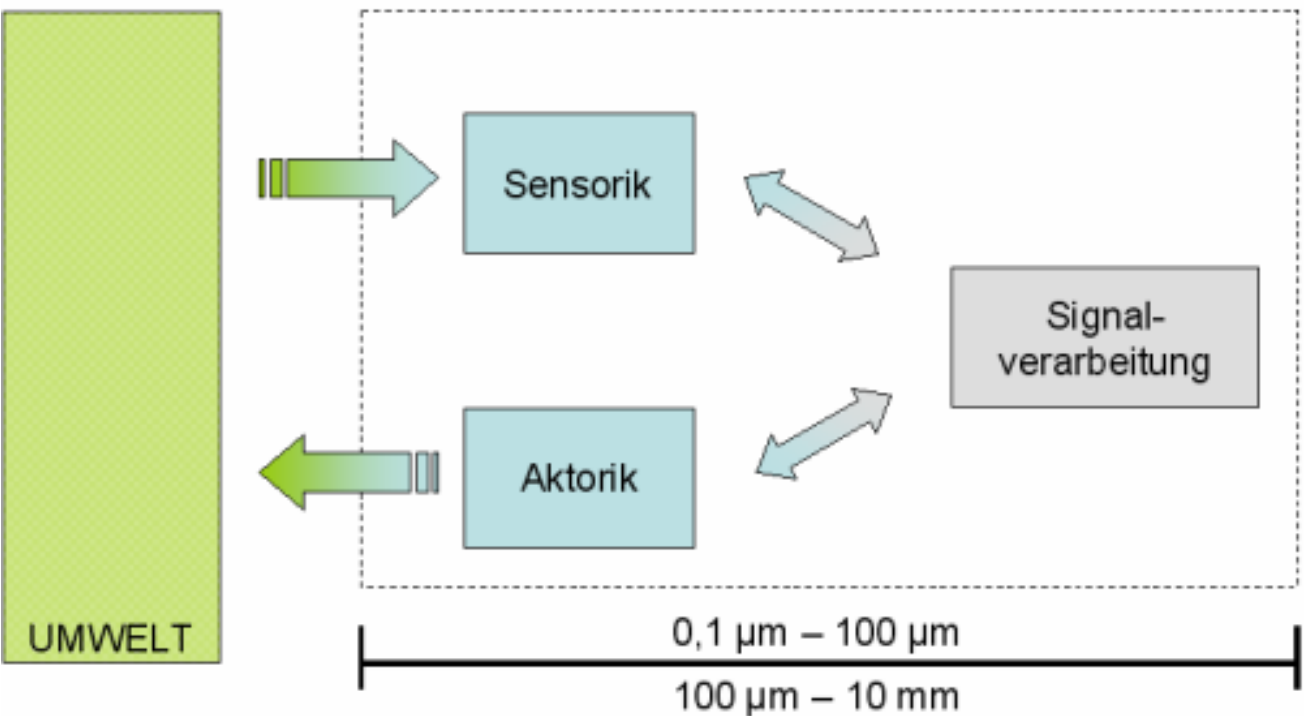

Abbildung 1: Definition „Mikrosystemtechnik“ (nach BARK)

Es sei kurz angemerkt, dass für den anglo-amerikanischen Raum die Bezeichnung MEMS (Micro-ElectoMechanical Systems) gebräuchlich ist, und die Verbindung von mechanischen und elektrischen Komponenten im Mikrometer- bis Millimeterbereich bezeichnet.

\subsection{Rahmenbedingungen der MST}

Aufgrund der geringen Größe wird die Funktionsfähigkeit der Systeme meist bereits durch kleinste Schmutzpartikel beeinträchtigt. Deshalb gelten für Fertigung und Montage häufig besondere Anforderungen an die Sauberkeit der Luft. Um dem Rechnung zu tragen, werden Reinräume in unterschiedliche Klassen - gemäß DIN 14644 - eingerichtet. In ihnen werden Kontaminationsquellen vermieden und der Luftstrom kontinuierlich gefiltert und überwacht, was mit einem hohen Energieaufwand verbunden ist. Über eine Schleuse werden die Bauteile in die Reinraumatmosphäre verbracht.

Eine weitere Störquelle sind elektro-statische Aufladungen, die wie ein Blitzschlag auf das fragile Bauteil einwirken, und es sofort zerstören. Deshalb müssen sowohl Handhabungsgerät und Arbeitsflächen als auch Ladehilfsmittel elektro-statisch geschützt werden.

\subsection{Wirtschaftliche Bedeutung}

Vor allem immer mehr kleine und mittelständische Unternehmen (KMU) nutzen die Mikrosystemtechnik mit der in Deutschland etwa 680.000 Arbeitsplätze unmittelbar verknüpft sind [BMBF0]. Viele Produkte wären ohne die MST-Komponenten gar nicht zu verkaufen, wodurch die (wirtschaftliche) Bedeutung noch weiter steigt („Leverage Effekt“). So wird der Umsatz, mit Produkten in denen MST integriert ist, für die deutsche Industrie auf $€ 270$ Mrd. geschätzt.

Fast unbemerkt erhielt die Technik Einzug in die Automobilindustrie, Medizin-/Life Science-Technik, Umwelttechnik, Logistik, Maschinen- und Anlagenbau und noch viele andere Bereiche. 
Der weltweite Markt für komplette Mikrosysteme betrug 2005 etwa US-\$ 68 Mrd. und Schätzungen gehen davon aus, dass der Markt bis 2010 noch auf über 200 Mrd. ansteigen wird.

Allein in Deutschland wurde bereits im Jahr 2000 mit MST-Komponenten und kompletten Systemen ein Umsatz von $€ 4,2$ Mrd. generiert. Schätzungen des VDE gehen von einem überdurchschnittlichen, jährlichen Wachstum von 17 \% für Produkte die mikrosystemtechnische Lösungen enthalten aus. Der NEXUS task force report „Market Analysis for Microsystems (2000 - 2005)“ spricht sogar von einem jährlichen Wachstum von 20 \%. Damit zählt die Mikrosystemtechnik zum entscheidenden Innovationstreiber für die deutsche Wirtschaft.

\section{Der Einsatz von Materialflusstechnik in Unternehmen der MST}

\subsection{Größeneffekte}

Fertigungsverfahren und Materialflusstechnik sind eng vernetzt und beides wird im konventionellen Bereich sehr gut beherrscht. Mit der Notwendigkeit der Herabskalierung von Bauteildimensionen für die MST, waren die Fertigungsverfahren zuerst konfrontiert. Zum Teil konnte die vorhandene Kompetenz konventioneller Fertigungsverfahren nach DIN 8580 genutzt werden (Mikrozerspanung, Mikrosintern, u.a.), oder es wurden Verfahren speziell für die Mikrosystemtechnik entwickelt, wie z.B. das LIGA-Verfahren (TiefenlithografieGalvanik-Mikroabformung) für die Erzeugung dreidimensionaler Strukturen. Jedoch konnten die Verfahren nicht beliebig herunterskaliert werden. Unterschreitet man (je nach Verfahren) eine gewisse Größe, so kehren sich plötzlich Größenverhältnisse um, und es dominieren (Stör-)Kräfte, die vorher aufgrund des größeren Gewichts noch nicht relevant waren. Die Fertigungstechnik musste zuerst auf diese Effekte reagieren [Wulfsberg05].

Im gleichen Maße kommt es zu Größeneffekten die sich auf die Technische Logistik auswirken, und von der Mikrologistik näher untersucht werden. Die einzelnen Effekte werden im Folgenden kurz dargestellt:

Adhäsionskräfte - Die wohl wichtigste Auswirkung die das Bauteil erfährt sind die steigenden Adhäsionskräfte, in Form der van-der-Wals Kraft und der elektrostatischen Kraft (vgl. Abb. 2). Durch das relativ geringe Eigengewicht löst sich das Bauteil nicht mehr von alleine von der Berühroberfläche, und bleibt haften.

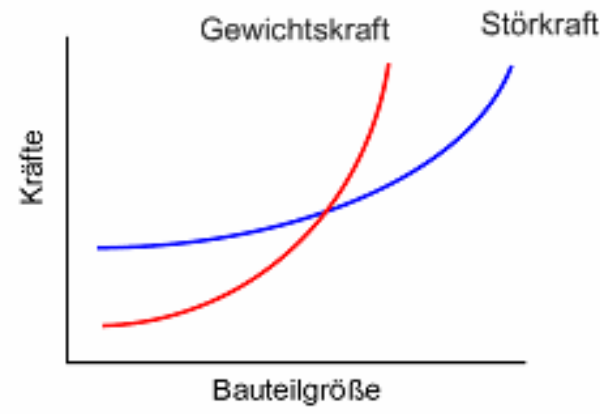

Abbildung 2: Relevante Kräfte in Abhängigkeit von der Bauteilgröße

Die Adhäsionskräfte sind vom Abstand abhängig und nehmen mit größer werdender Distanz quadratisch ab. Durch eine entsprechende Beschichtung wird versucht diese Kräfte möglichst gering zu halten. In der Handhabungstechnik wird versucht eine zur Haltekraft umgekehrte Kraft aufzubringen. Das kann bei einem Sauggreifer z. B. mit einem Luftstoß geschehen. Wenn die Adhäsionskräfte nicht kontrolliert werden können, wird es schwierig das Teil zu positionieren und $\mathrm{zu}$ orientieren, was jedoch Voraussetzung für eine Automatisierung ist. Außerdem besteht das aus der Mikroelektronik schon seit längerem bekannte Problem der elektrostatischen Entladung, welche die Halbleiterbauelemente zerstört. Deshalb ist ein Schutz vor elektrostatischen Entladungen (ESD-Schutz / Electro Static Discharge) unverzichtbar.

Genauigkeit - Kleinere Bauteilstrukturen erfordern höhere Genauigkeiten in der Handhabung. Je kleiner die Elemente werden, desto präziser muss die Handhabungstechnik sein, die die Bauteile aus der Fertigung übernimmt, um sie dem weiteren Materialfluss zu zuführen. Die Positioniergenauigkeit reicht, in Abhängigkeit von der Aufgabe, von $20 \mu \mathrm{m}$ bis hinunter zu etwa 0,1 $\mu \mathrm{m}$. Die Herausforderung tritt dann auf, wenn die herkömmliche Technik nicht mehr die geforderte Genauigkeit leisten kann. Dann muss eine komplett neue Technik zum Einsatz kommen. Beispielsweise sind konventionelle 
Zwei-Backengreifer für ein großes Spektrum an Handhabungstätigkeiten geeignet, wachsen jedoch die Anforderungen an die Positioniergenauigkeit stark an, dann haben Piezo-Aktoren den Vorzug.

Identifikation - Ist der Transport oder die Lagerung eines Teils nicht hinreichend dokumentiert, so kommt es mitunter zu Schwierigkeiten, ein Bauteil eindeutig zu identifizieren. Ohne mikroskopische Hilfsmittel ist es meist unmöglich zu erkennen, ob ein Zahnrad $500 \mu \mathrm{m}$ im Durchmesser misst oder $700 \mu \mathrm{m}$, und somit die gewünschten Teile zu entnehmen. Deswegen kommt der vollständigen Dokumentation der Bauteileigenschaften, und einer eindeutigen Zuordnung der Dokumente während es sich im Materialflussprozess befindet, eine hohe Bedeutung zu. Die falsche Zuordnung von Begleitpapieren und Bauteil wird häufig nicht erkannt, und der Fehler fällt erst in der Abschlusskontrolle auf, was eine sehr teure und aufwendige Fehlerbeseitigung bedeutet.

Materialkosten - Die gleichzeitige Herstellung von mehreren Millionen Transistoren mit einer Charge von Wafern („Batch“), hat die Herstellkosten wegen der erhöhten Packungsdichte schlagartig um Zehnerpotenzen gesenkt. Dieses war eine direkte Folge der konsequenten Verringerung der Größe auf dem Gebiet der Mikroelektronik. Dieses Beispiel verdeutlicht, dass insbesondere die Materialkosten von einer weiteren Miniaturisierung profitieren, wenn gleich die Qualitätsanforderungen an das eingesetzte Material ansteigen.

Energieverbrauch - Mikrosysteme besitzen i.d.R. drei von einander getrennte Komponenten: eine Sensorik, eine Signalverarbeitung um die erfassten Daten auszuwerten und eine Aktorik. Um gezielt den Energieverbrauch zu reduzieren, sind Entwickler und Konstrukteure bemüht, die Miniaturisierung der Komponenten voranzutreiben. Die geringen Abmaße und die kurzen Leiterbahnen verringern den Energiebedarf entscheidend, was u. U. einen Marktvorteil darstellt. Neuere Forschungsvorhaben arbeiten an einer Sensorintegration in den Aktor, wodurch der Energiebedarf - zusammen mit einem intelligenten Energiemanagement - noch weiter reduziert werden kann.

Bauraum - Häufig werden Mikrosystemkomponenten in andere Produkte integriert und bilden in ihnen wesentliche Funktionen ab, ohne die das Produkt selber meist nicht denkbar wäre. Durch den geringen Bauraum und das geringe Gewicht, wird es erst möglich bestimmte Geräte mobil einzusetzen (z.B. einen Gaschromatographen). Wichtigster Markt für Unternehmen der MST in Deutschland ist mit $38 \%$ die Medizintechnik (Abb. 3), wozu auch der Bereich der Life Sciences zählt [IVAM05]. In Analyse- und Diagnosegeräten, aber auch in Endoskopen für die minimal invasive Chirurgie, nutzt man die geringe Baugröße und das geringe Gewicht.

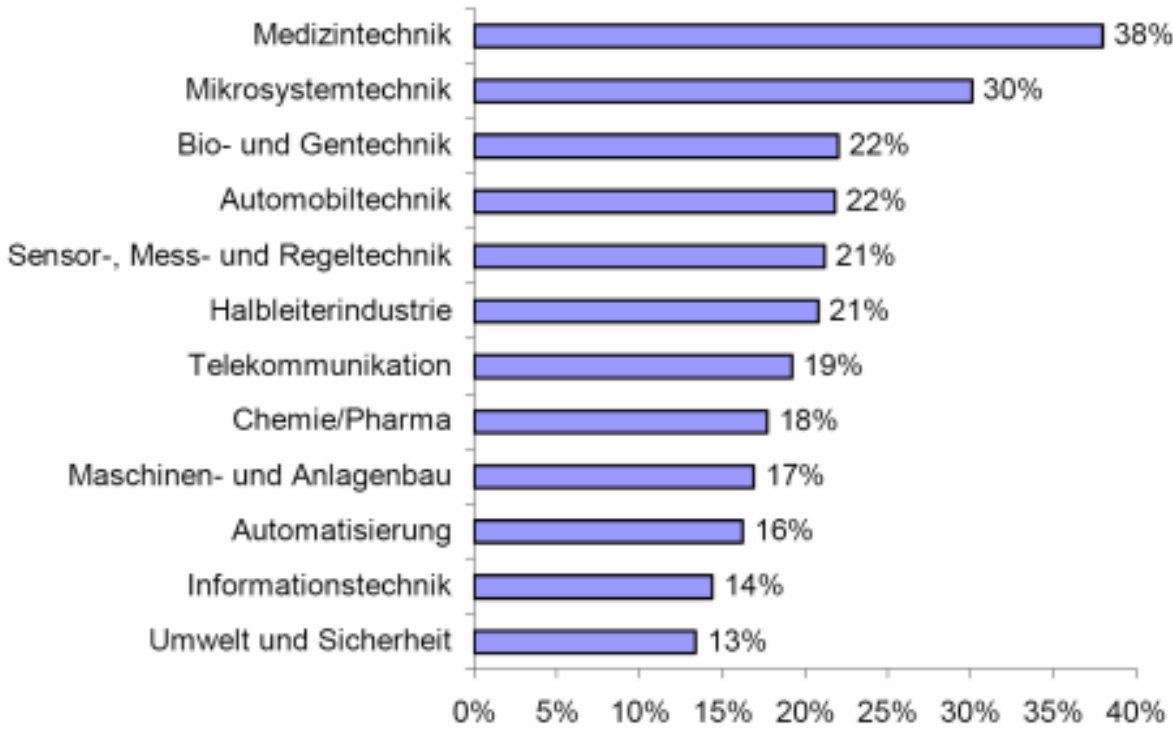

Abbildung 3: Zielmärkte für deutsche Unternehmen der MST (Quelle: IVAM)

Für das Unternehmen wirkt sich der geringe Bauraum vorteilhaft auf die Produktionslogistik aus: Puffer und Läger müssen weit aus kleiner dimensioniert werden und es muss weniger Lagerfläche zur Verfügung gestellt werden, als für die Fertigung von Produkten aus der Makrowelt. 


\subsection{Fördertechnik}

Für den innerbetrieblichen Transport zwischen den Fertigungsprozessen, bzw. ins Lager, werden Stetigförderer und Unstetigförderer eingesetzt. Die am häufigsten eingesetzten Stetigförderer sind Gurtbandförderer, die dem Bandförderer angelehnt sind, und bis zu einer Breite von 15 mm herunterskaliert werden. Die Förderstrecke beträgt wenige Meter und die Bandgeschwindigkeit beträgt bis zu 1,5 m/s. Als Trag- und Zugorgan kommen öl-, fett- und emulsionsbeständige Kunstoffbänder zum Einsatz, auf denen das Teil transportiert wird. Soll das Teil auf Ladehilfsmitteln gefördert werden, so eignen sich hierfür ein Eingurtband mit Stützrollen oder ein Doppelgurtband. Das Eingurtband mit Stützrollen ist eine Kombination aus dem Eingurtförderer, der als antreibender Transportstrang dient, und einem parallel verlaufenden Strang mit Stützrollen. Das Doppelgurtband besteht aus zwei stetig umlaufenden, prallelen Gurten. Mit beiden Fördermitteln können lose aufliegende Ladehilfsmittel mit Geschwindigkeiten bis zu 0,3 m/s gefördert werden. Die Spurbreite lässt sich leicht an das Ladehilfsmittel anpassen; üblich sind Werte zwischen $120 \mathrm{~mm}$ und $800 \mathrm{~mm}$, sowie Längen zwischen $2 \mathrm{~m}$ und 50 m. Ebenfalls geeignet für den Einsatz in der Mikrosystemtechnik sind Gliederbandförderer. Sie gehören zur Gruppe der Kettenförderer und haben eine Segmentkette als Tragorgan, mit der horizontale und vertikale Kurven auf engstem Raum realisiert werden können. Die Segmentkette kann eine Breite von minimal 40 mm und eine Länge von bis zu $30 \mathrm{~m}$ besitzen. Die Fördergeschwindigkeit beträgt etwa $1 \mathrm{~m} / \mathrm{s}$.

Wenn es um das vereinzeln von Schüttgut in Stückgut geht, sowie um das eindeutige positionieren und orientieren von Teilen für die automatisierte Montage, dann kommt häufig eine Wendelschwingrinne zum Einsatz. Sie ist eine besondere Bauform der Vibrationsförderer zu denen u.a. die Schwingrinne gehört. Das Fördergut erfährt zunächst eine vertikale Beschleunigung, wodurch es aufgrund der Massenträgheit abhebt, und eine anschließende horizontale Beschleunigung, die das Teil weiterfördert. Aufgrund der Mikrosprünge können Wendelschwingrinnen auch für den Vertikaltransport genutzt werden.

Für das Gebiet der Unstetigförderer werden die aus der Makroebene bekannten Flurförderzeuge für den Palettentransport eingesetzt. Innovative Lösungen für den Bereich der Greifertechnik werden in Kapitel 3.3 vorgestellt.

\subsection{Handhabungstechnik}

Je nach Anwendungsfall existieren sehr spezielle Handhabungstechniken. Insbesondere die Greifertechnik nutzt unterschiedlichste physikalische Prinzipien für die Positionierung und Orientierung von Bauteilen, die Informationsbereitstellung durch eine entsprechende Sensorik, die Teilebereitstellung bei der Montage oder die Reinigung in Fertigungsprozessen [Oh98]. Bei den taktilen Greifern kommen mit einem Anteil von über 80 \% überwiegend Vakuumgreifer und Mechanische Greifer (Finger-, Klemm- und Zangengreifer) zum Einsatz. Der Durchmesser des Saugnapfes eines Vakuumgreifers ist auf 0,5 mm begrenzt. Zur Handhabung kleiner Teile, verwendet man Saugrohre mit einer Glasplatte an der Unterseite, die mit einer Saugstruktur versehen ist. Eine Venturi-Düse im inneren des Ejektors sorgt für den nötigen Unterdruck. Bei den Zangengreifern unterscheidet man nach der Art des Antriebes. Der Greiferfinger kann über einen Hitzdrahtantrieb, Piezoantrieb, elektromotorischen Antrieb oder einer Kombination zweier Antriebe bewegt werden, um möglichst genau, und mit der notwendigen Greiferkraft, das Mikroteil zu handhaben.

Adhäsionsgreifer hingegen stellen eine stoffschlüssige Verbindung mit dem zu transportierendem Teil her, manipulieren es, und lösen es wieder vom Greifer. Dieses kann z.B. mit Hilfe eines Wassertropfens als Adhäsiv geschehen. Ein völlig anderes Wirkprinzip verfolgen Adhäsionsgreifer mit einem getaktetem Klebeband. Der Kleber hält das Teil wenn es aufgenommen wird, und eine Nadel löst das Teil, sobald es abgelegt werden soll. Anschließend wird das Klebeband weitergetaktet. Nachteilig ist allerdings die Verschmutzung die durch das Klebeband auf dem Bauteil zurückbleibt.

Ganz ohne Berührung arbeiten hingegen Ultraschall-Vakuumgreifer und Luftkissengreifer. Sie arbeiten berührungsfrei und vermeiden dadurch Beschädigungen am Bauteil. Sie werden allerdings noch nicht in der Industrie eingesetzt, sondern durch den Forschungsverbund „ForMikroProd“ weiterentwickelt [Zäh05].

\subsection{Lagertechnik}

Gerade kleine Unternehmen greifen bei der Lagertechnik eher zu unkonventionellen Lösungen, wobei dem Erfindungsreichtum für eine „Fremdverwendung“ keine Grenzen gesetzt sind. Die eingesetzte Lagertechnik ist häufig an Büroartikeln angelehnt, wie z.B. Schubladenboxen im DIN-Format. Sie eignen sich hervorragend für die kleinen Teile der Mikrosystemtechnik. Zur Aufnahme der Ladehilfsmittel (z.B. Wafer-Box oder Lager-FixKästen) kommen typische Fachbodenregale zur Anwendung, wie sie bereits hinreichend in der Makroebene zur (statischen) Lagerung von Stückgütern eingesetzt werden.

Umlaufregale, in horizontaler und vertikaler Ausführung, eignen sich für die dynamische Lagerung von Kleinteilen, und somit auch für Teile der MST. Bei der Ein- und Auslagerung wird die gesamte Regalzeile durch einen Stetigförderer bewegt, und die Güter werden aus den einzelnen Fächern manuell entnommen. Ein anderes dynamisches Lagermittel ist der Lifter. 
Die einzelnen Tablare können Kästen aufnehmen, die mit MST-Komponenten oder anderen Kleinteilen gefüllt sind. Die Tablare sind als Regalfächer an der Vorder- und Rückseite des Lifters angeordnet. Dadurch, dass der Positionierlift die Regalhöhe für die Einlagerung frei wählen kann, besteht eine optimale Raumausnutzung.

\section{Zusammenfassung und Ausblick}

Die technische Logistik steht für Unternehmen der Mikrosystemtechnik noch am Anfang. Häufig sind die Stückzahlen so gering, dass ein hoher Automatisierungsgrad sich noch nicht lohnt. Es ist jedoch zu erwarten, dass bei weiteren hohen Wachstumsraten in der Mikrosystemtechnikbranche, die Ansprüche an die Fördertechnik steigen werden. Die Studie hat ergeben, dass vielfach Insellösungen in Unternehmen vorherrschen und eine einheitliche, systematische Vorgehensweise bei der Analyse und Planung, speziell für die Besonderheiten der MST, nicht existiert.

Wertet man die eingesetzten Materialflussmittel aus, so lassen sie sich in drei Klassen einteilen:

Klasse I: Materialflussmittel wurden unverändert aus der Makroebene übernommen. Das trifft für Kästen, Rollbehälter, Fachbodenregale u. a. zu. Dieses wird dadurch ermöglicht, dass die Mikrosysteme mit Ladehilfsmittel verbunden werden, und somit wie Kleinteile gehandhabt werden können.

Klasse II: Existierende Lösungen aus der Makroebene werden herunterskaliert bzw. für die MST angepasst. Als Beispiel wären hier der Zweibackengreifer und die Gliederbandförderer zu nennen.

Klasse III: Vorhandene, artfremde Lösungen werden auf die MST übertragen und im Materialfluss eingesetzt. Dieses trifft für die Lagermittel zu, die sich bei Lösungen aus dem Büroartikelbereich bedient.

Zukünftige Arbeiten am Lehrstuhl MTL werden sich damit befassen, wie die Mikrosystemtechnik, unter Berücksichtigung der erläuterten Größeneffekte, zweckmäßig in die Supply Chain integriert werden kann. Ziel ist es ein ganzheitliches logistisches Konzept für den Übergang von der Makro- in die Mikroebene zu entwickeln.

\section{Literatur}

[Bark99]

[BMBF06]

[Gerlach97]

[Hesselbach02]

[IVAM05]

[Menz05]

[Mescheder04]

[Oh98]

[Wulfsberg05]

[Zäh05]
Bark, K.-B.: Adhäsives Greifen von kleinen Teilen mittels niedrigviskoser Flüssigkeiten. Berlin (usw.): Springer, 1999 Bundesministerium für Bildung und Forschung: Mikrosystemtechnik, http://www.bmbf.de/de/5701.php (Datum des letzten Zugriffs: 26. Mai 2006) Gerlach, G., Dötzel, W.: Grundlagen der Mikrosystemtechnik. München: Hanser, 1997 Hesselbach, J.: mikroPRO - Untersuchung zum internationalen Stand der Mikroproduktionstechnik. Essen: Vulkan-Verlag, 2002

IVAM Research: MST-Atlas Deutschland/ Mikrosystemtechnik-Cluster in Deutschland, Ausgabe 2005, Dortmund: IVAM Research 2005

Menz, W., Mohr, J., Paul, O.: Mikrosystemtechnik für Ingenieure. Weinheim: Wiley-VCH Verlag, 2005

Mescheder, U.: Mikrosystemtechnik - Konzepte und Anwendungen. Wiesbaden: Teubner Verlag, 2004 Oh, Hyeon-Seok: Elektrostatische Greifer für die Mikromontage. Düsseldorf: VDI Verlag, 1998

Wulfsberg, J.-P., Terzi, M.: Prägende Momente. IN: Mikroproduktion (2005) 3 Zäh, M. et al. : Aus der Luft gegriffen. IN: Mikroproduktion (2005) 1 